\title{
Feasibility and effectiveness of remotely supervised tDCS combined with computerized cognitive training for patients with stroke: A randomized sham-controlled study
}

\author{
Ju-Yul Yoon \\ Mi-Nam Son \\ Sungkyunkwan University School of Medicine \\ Yun-Ju Jo \\ Jeonbuk National University Hospital \\ Da-Sol Kim \\ Jeonbuk National University Medical School

\section{Gi-Wook Kim} \\ Jeonbuk National University Medical School \\ Yu-Hui Won \\ Jeonbuk National University Medical School \\ Sung-Hee Park \\ Jeonbuk National University Medical School \\ Jeong-Hwan Seo \\ Jeonbuk National University Medical School \\ Yun-Hee Kim \\ Sungkyunkwan University School of Medicine \\ Myoung-Hwan Ko ( $\square$ mhko@jbnu.ac.kr) \\ https://orcid.org/0000-0002-0566-3677
}

Jeonbuk National University Medical School https://orcid.org/0000-0002-2379-6158

\section{Research}

Keywords: Cognitive dysfunction, Telerehabilitation, Stroke, Transcranial direct current stimulation, tDCS, Non-invasive brain stimulation

Posted Date: January 26th, 2021

DOI: https://doi.org/10.21203/rs.3.rs-146962/v2

License: @ (i) This work is licensed under a Creative Commons Attribution 4.0 International License. Read Full License 


\section{Abstract}

Background Cognitive impairment after stroke is an unfavorable factor for long-term functional independence. Transcranial direct current stimulation (tDCS) is a promising tool for improving cognitive function in patients with stroke. Home-based rehabilitation is increasingly required for patients with stroke, with greater benefits expected if supplemented with remotely supervised tDCS (RS-tDCS). We evaluated cognitive improvement and the feasibility of RS-tDCS in patients with chronic stroke.

Methods Thirty chronic stroke patients with cognitive impairment (K-MoCA <26) received a computerized cognitive training package (Comcog ${ }^{\mathrm{TM}}$, Neofact, Seoul, Korea), and were randomized into real RS-tDCS and sham RS-tDCS groups according to the application of tDCS. Participants were treated 5 days/week for 4 weeks. To ensure correct self-application of tDCS (Mindd Stim ${ }^{\circledR}$, Ybrain Inc., Korea), patients and caregivers received training and were monitored. Several cognitive evaluations were performed. Rate of adherence to the appropriate RS-tDCS session was also investigated.

Results Among the 30 participants, 2 chose to withdraw and 2 were excluded due to noncompliance. In the within-group comparison, unlike the sham group $(n=14)$, the real group $(n=12)$ showed a significant improvement in K-MoCA (intra- $p=0.004$ vs. 0.132$)$, particularly in patients with moderate cognitive impairment (K-MoCA: $10-17$; intra- $p=0.001$ vs. 0.835 , K-MoCA: $18-25$; intra- $p=0.060$ vs. 0.064). However, in K-DRS2, Stoop and K-BNT, both groups showed significant improvement, but there was no group time interaction. In the Trail Making Test, Go/no Go, and Controlled Oral Word Association Test, both groups did not show statistically significant improvement. A total of 551 of the 560 sessions conducted by 28 people were successfully performed (adherence rate: $98.4 \%$ ) and no serious adverse effects were detected.

Conclusion RS-tDCS is a safe and feasible rehabilitation modality for post-stroke cognitive dysfunction.

It has the potential to enhance the effect of home-based CT. RS-tDCS seems to be particularly effective in patients with relatively more severe cognitive impairment who are capable of home-based training.

Trial registration: Clinical Research information Service (KCT0003427). Registered 26 June 2018, https://cris.nih.go.kr/cris/en/search/search_result_st01.jsp?seq=12363

\section{Background}

When cognitive dysfunction after stroke persists, it is known to be an unfavorable factor for long-term functional independence [1]. There have been a number of studies on the effects of transcranial direct current stimulation (tDCS) in relation to cognitive dysfunction. It has been reported that tDCS can enhance working memory in healthy people and prevent cognitive dysfunction in the elderly $[2,3]$.

Moreover, tDCS is effective at improving cognitive function in several pathologies, including Alzheimer's and schizophrenia, and major depressive disorder [4,5]. tDCS has also been reported to not only improve cognition, including working memory [4-7], but also neglect syndrome $[4,8,9]$ and stroke-related depression [4]. In particular, several studies have demonstrated the effect of improving post-stroke aphasia $[4,10]$.

Recent research trends have focused on the convenience, feasibility, and effectiveness of telerehabilitation [11]. It has been reported that patients with stroke show a decline in function after discharge from inpatient rehabilitation facilities [12]. Due to the pressure at rehabilitation institutions to reduce the length of inpatient stay, the rehabilitation treatment environment is frequently changing, and it is difficult to form a suitable, familiar environment for patients [13].

In rural areas, it is difficult to continue outpatient cognitive training (CT) through outpatients due to limited accessibility [11]. Furthermore, medical institutions visits are decreasing due to infectious diseases such as coronavirus disease 2019.

Since computerized cognition treatment is feasible at home [14], if patients are also able to safely and appropriately use tDCS at home, it would be possible to increase the effectiveness of cognitive therapy. Depending on the degree of supervision and intervention by supervisors, home-based application of tDCS can be divided into home-use tDCS, remotely supervised tDCS (RS-tDCS), and remotely controlled tDCS [15]. In home-use tDCS, patients can control the mode or application time themselves and use the device without supervision. In RS-tDCS, patients cannot control the tDCS settings and receive online support from a supervisor to administer duration and intensity. In remotely controlled tDCS, the tDCS device is always monitored by the supervisor and can only be controlled by the supervisor [15]. However, ethical issues have been raised regarding the unlimited use of tDCS. A certain level of intervention is required 
to reduce the possibility of misuse and abuse and for optimal application of tDCS $[4,16]$. Therefore, for efficiency and safety, RS-tDCS might be the most suitable option.

In studies applying home-based rehabilitation with RS-tDCS in patients with multiple sclerosis and Parkinson's disease, groups receiving RS-tDCS plus CT showed greater improvements in cognitive function than groups that only received CT [17, 18]. Thus, RStDCS has been demonstrated to be a feasible treatment strategy. To date, there have been few studies on home-based application of tDCS in patients with stroke [11], and research on its effectiveness and feasibility is needed.

To the best of our knowledge, there are no previous studies investigating the effectiveness of RS-tDCS at improving cognitive function in patients with stroke. In this pilot study, we aimed to explore whether there was a cognitive enhancing effect when tDCS was combined with home-based CT and to investigate the feasibility of RS-tDCS for patients with chronic stroke.

\section{Methods}

\section{Study design}

This study was a double-blind sham-controlled randomized trial, approved by the Samsung Medical Center Ethics Committee and registered with Clinical Research information Service (KCT0003427) and conducted in the two stroke centers (Samsung Medical Center, Jeonbuk National University Hospital).

\section{Participants}

Participants were aged 20-85 years with chronic stage ( $\geq 6$ months after onset) stroke, including ischemic and hemorrhagic stroke, with cognitive dysfunction (Korean version of the Montreal Cognitive Assessment [K-MoCA] score <26), who voluntarily decided to participate, signed the consent form, and were capable of responding to a questionnaire. When a patient was deemed to have difficulty self-administering tDCS, they were only included if they had a caregiver capable of administering tDCS regularly. The exclusion criteria were patients with an existing severe neuropsychiatric disorder (such as schizophrenia, bipolar disorder, or degenerative dementia), contraindication for tDCS (patients with implanted medical devices, metallic cranial implants, skin wound at electrode placement sites, history of epilepsy, and problems with electrode attachment, such as dermatological issues), severe cognitive impairment (K-MoCA $\leq 9)$ restricting their ability to understand cognitive tasks, and pregnancy.

\section{Randomization}

At baseline, eligible participants were assigned a participant registration number by the investigator. The order for medical device allocation was randomized by participant registration number using GraphPad software (http://www.graphpad.com/quickcalcs/index.cfm) performed by a third party other than the investigator.

\section{Intervention}

The two groups underwent 20 sessions of sham or real tDCS (Mindd Stim ${ }^{\circledR}$, Ybrain Inc., Korea) plus CT. Participants participated in 5 sessions per week for 4 weeks; each session lasted 30 min (Fig. 1).

\section{Transcranial direct current stimulation}

Before applying RS-tDCS, the guidelines of Charvet et al. were followed [19]. At baseline assessment (T0), participants received tDCS procedure instructions and performed a trial application. Tolerability was checked, and if the participants had caregivers, the caregivers also received identical instructions for the tDCS procedure. The entire process of applying the equipment was recorded on the participant or caregiver's smartphone for reference during later home-based application. After receiving instructions, the participant or caregiver was asked to repeat the whole application process by themselves until they were able to complete the process properly. Once the participant had successfully performed the trial application, they were provided with the RS-tDCS devices and instructional manual.

At the first home-based RS-tDCS session, the research physician observed whether it was properly performed by the participant. If the participant required further instruction, the process was repeated until the participant or caregiver could independently perform the process. The research physician also determined whether the participant's home was a suitable environment to perform RS-tDCS plus CT. 
At the remote sessions, the participants were monitored by telephone 3 times per week. If appropriate communication was difficult on the phone, monitoring was performed via a video call. The tDCS devices were set in advance to allow 1 stimulation per day, and additional use was not permitted. The duration, intensity, and impedance were logged so that the investigators could check for adequate compliance after all remote sessions. During the 20 sessions, the participants visited the hospital once per week to check the tDCS device operation status and the adequacy of tDCS application was inspected.

Regarding electrode location, the anode was placed over the left dorsolateral prefrontal cortex (DLPFC) as determined by the International 10/20 Electroencephalogram System corresponding to F3, and the cathode was placed in the right supraorbital area. Participants were provided with $28.3 \mathrm{~cm}^{2}$ disposable electrodes and were instructed to soak them in $20 \mathrm{ml}$ saline before use. A head cap was used to standardize the electrode location; the positions were fixed at Visit 1, so that the electrodes would be in the p position when the participant wore the cap on their head in the correct orientation (Fig. 2).

A constant current of $2 \mathrm{~mA}$ was administered for $30 \mathrm{~min}$. The electrode placement was identical for the sham tDCS stimulation; however, the stimulator was turned on for only 10 seconds, during which the current intensity gradually increased and then decreased until it diminished. The allocation of tDCS was blinded to investigators, research physicians, participants, and caregivers.

\section{Cognitive training}

For the computerized CT program, Comcog ${ }^{\mathrm{TM}}$ (v.2.6, Neofact, Seoul, Korea) was installed on a 21-in tablet and provided to each participant at home [20]. The program consists of memory, attention, executive function, and spatiotemporal perception training, and all participants were instructed to train with the same composition for $30 \mathrm{~min}$ per session. Difficulty was adjusted differently for each participant. At Visit 1, a physician guided participants to use the appropriate CT package, then the participants visited the hospital once per week to review the previous week's training results and receive a new training task adapted to their level. Participants received tDCS simultaneously for $30 \mathrm{~min}$.

\section{Safety}

The tDCS parameters used in this study did not cause severe adverse effects in previous research [21]. In this study, the devices were set to enable only 1 session of tDCS per day, and an appropriate impedance $(<7,000 \Omega)$ range was set during module application. The RS-tDCS hub recorded impedance logs, which could be used to check safety and monitor protocol adherence. In addition, participants were contacted by phone 3 times per week to check abnormal symptoms.

\section{Outcome measures}

Outcomes data were collected by the Jeonbuk National University Hospital Clinical Research Center and Samsung Medical Center at baseline and post-intervention.

\section{Primary outcomes}

\section{Standard cognition tests}

The K-MoCA was used to assess overall cognitive function. MoCA has been used to assess cognitive function in several previous tDCS studies [22] and is considered an effective tool to conveniently assess cognitive function [23]. The K-MoCA was previously validated with the MoCA [24]. The Korean version of the Dementia Rating Scale-2 (K-DRS-2) was used as a second tool to assess overall cognitive function [25].

\section{RS-tDCS adherence rate}

To investigate RS-tDCS feasibility, the completion rate and protocol adherence were monitored, and feedback on problems while performing the intervention was received.

\section{Secondary outcomes}

The Stroop word test, trail making test (TMT), Go/no Go test, Controlled Oral Word Association Test (COWAT) and Korean-Boston Naming Test (K-BNT) were used to evaluate the cognition of each sub-territory. COWAT, TMT A B, BNT, and K-BNT were performed 
based on the Seoul Neuropsychological Screening Battery [26]. The Stroop word test was performed based on the Computerized NeuroCognitive Function Test software-40 (Maxmedica Ltd., Seoul, Korea) [27].

Statistical analysis

Participants were assessed at baseline before treatment (T0), in the primary assessment within 48 hours of completing treatment ( $\mathrm{T} 1$ ), and in the secondary assessment approximately 1 month after treatment (T2). Analysis of covariance (ANCOVA) was used to examine whether there were significant differences between the sham and real groups at T1 and T2 when controlling for assessment outcomes at TO. If normality was not satisfied in the between-group comparison, ranked ANCOVA was used. Repeated measures-analysis of variance (RM-ANOVA) was used to examine differences within each group. If the normality was not satisfied in the within-group comparison, the Friedman test was used. Statistical significance was defined as $p<0.05$. Statistical analyses were performed using IBM SPSS 24 (SPSS Inc., Chicago, IL, USA).

\section{Results}

A total of 30 participants were enrolled, but 2 were excluded due to withdrawal of consent and 2 were excluded due to noncompliance with multiple remote sessions. Therefore, 26 participants were included in the final analysis (Fig. 3); their demographic data are shown in Table 1.

Table 1. Demographic characteristics of participants

\begin{tabular}{|c|c|c|c|c|}
\hline Variable & Real RS-tDCS plus CT $(n=12)$ & Sham $R S$-tDCS plus CT $(n=14)$ & Total $(n=26)$ & $p$-value \\
\hline Age & $61.25 \pm 12.85$ & $57.86 \pm 10.04$ & $59.42 \pm 11.32$ & $0.470^{\mathrm{a}}$ \\
\hline Gender & & & & $0.229^{\mathrm{c}}$ \\
\hline Male & $4(33.3)$ & $8(57.1)$ & $12(46.2)$ & \\
\hline Female & $8(66.6)$ & $6(42.9)$ & $14(53.8)$ & \\
\hline Education period (year) & $13.08 \pm 5.38$ & $11.36 \pm 4.62$ & $12.15 \pm 4.96$ & $0.200^{\mathrm{b}}$ \\
\hline K-MoCA & $18.75 \pm 5.19$ & $21.00 \pm 4.13$ & $19.96 \pm 4.69$ & $0.195^{\mathrm{b}}$ \\
\hline Brain lesion side (Right : Left) & $3: 9$ & $7: 7$ & $10: 16$ & $0.184^{\mathrm{d}}$ \\
\hline \multicolumn{5}{|l|}{ BDNF } \\
\hline $\mathrm{G} / \mathrm{G}$ & $2(16.6)$ & $4(28.6)$ & $7(25.0)$ & \\
\hline $\mathrm{G} / \mathrm{A}$ & $6(50.1)$ & $8(57.1)$ & $15(53.6)$ & \\
\hline $\mathrm{A} / \mathrm{A}$ & $4(33.3)$ & $2(14.3)$ & $6(21.4)$ & \\
\hline \multicolumn{5}{|l|}{ APoE } \\
\hline E2 & $1(8.3)$ & $2(14.3)$ & $3(10.7)$ & \\
\hline E3 & $10(83.3)$ & $9(64.3)$ & $21(75.0)$ & \\
\hline E4 & $1(8.3)$ & $3(21.4)$ & $4(14.3)$ & \\
\hline
\end{tabular}

Data are $n(\%)$, mean \pm standard deviation

Statistical significance was defined as $p<0.05$

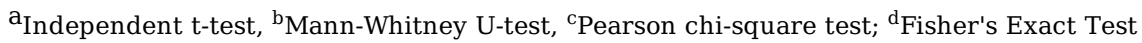

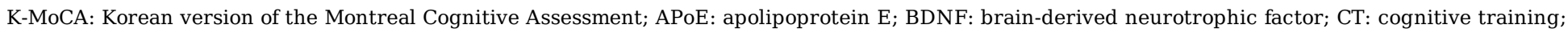

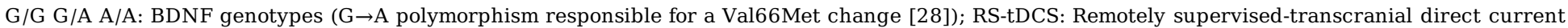
stimulation

\section{Primary outcomes}

\section{General cognition}

In the within-group comparison, the real group showed a statistically significant increase in K-MoCA scores $(p=0.01)$, but the sham group did not $(p=0.264)$. In contrast, in the between-group comparison, no statistically significant difference was found between groups (Table 2). After reanalyzing according to cognition degree, a statistically significant improvement was observed in participants with a moderate level of cognitive impairment (K-MoCA: 11-17), but not in those with a mild level of cognitive impairment (K-MoCA: 18-25) (Fig. 4). In the K-DRS-2, both groups showed a significant increase in the within-group comparison ( $p=0.002$ and $p=0.006$, respectively). In the between-group comparison, there was no statistically significant difference (Table 2).

Table 2. Results of cognitive function test and comparison between groups (Inter-p) and within groups (Intra- $p$ ) $(n=26)$ 


\begin{tabular}{|c|c|c|c|c|c|c|c|c|c|c|}
\hline & \multicolumn{4}{|c|}{ Real RS-tDCS plus CT $(n=12)$} & \multicolumn{4}{|c|}{ Sham RS-tDCS plus CT $(n=14)$} & \multirow{2}{*}{$\begin{array}{c}\text { T1 } \\
\text { Inter- } \\
p^{b)}\end{array}$} & \multirow{2}{*}{$\begin{array}{c}\text { T2 } \\
\text { Inter- } \\
p^{b)}\end{array}$} \\
\hline & Baseline & Post (T1) & Follow up (T2) & Intra- $p^{\text {a) }}$ & Baseline & Post (T1) & $\begin{array}{l}\text { Follow up } \\
\text { (T2) }\end{array}$ & Intra- $p^{\text {a) }}$ & & \\
\hline \multicolumn{11}{|l|}{ General cognition } \\
\hline K-MoCA & $18.67 \pm 5.16$ & $19.67 \pm 3.87$ & $21.33 \pm 4.42$ & 0.004 & $21.29 \pm 4.29$ & $22.07 \pm 4.95$ & $22.57 \pm 4.88$ & 0.132 & 0.762 & 0.464 \\
\hline K-DRS-2 & $120.00 \pm 12.08$ & $123.08 \pm 11.47$ & $124.00 \pm 11.44$ & 0.022 & $124.93 \pm 13.91$ & $128.43 \pm 12.96$ & $129.07 \pm 10.54$ & 0.003 & 0.583 & 0.458 \\
\hline \multicolumn{11}{|l|}{ Attention } \\
\hline \multicolumn{11}{|l|}{$\begin{array}{l}\text { Stroop (Reaction } \\
\text { time) }\end{array}$} \\
\hline Word & $24.29 \pm 8.20$ & $22.03 \pm 9.08$ & $21.02 \pm 9.89$ & 0.001 & $28.92 \pm 28.12$ & $20.53 \pm 11.97$ & $20.11 \pm 17.09$ & 0.002 & 0.487 & 0.204 \\
\hline Color & $43.90 \pm 26.77$ & $46.84 \pm 30.31$ & $40.38 \pm 23.67$ & 0.059 & $43.68 \pm 29.58$ & $34.13 \pm 22.07$ & $34.27 \pm 21.57$ & 0.011 & 0.107 & 0.167 \\
\hline Color word & $30.63 \pm 28.85$ & $31.60 \pm 29.15$ & $20.58 \pm 7.18$ & 0.040 & $27.79 \pm 28.21$ & $20.50 \pm 13.80$ & $19.45 \pm 42.60$ & 0.002 & 0.217 & 0.244 \\
\hline Word of color word & $33.94 \pm 28.48$ & $35.58 \pm 28.91$ & $26.58 \pm 11.26$ & 0.862 & $30.87 \pm 29.23$ & $24.45 \pm 15.99$ & $23.85 \pm 14.10$ & 0.232 & 0.163 & 0.709 \\
\hline Color of color word & $96.47 \pm 55.36$ & $113.86 \pm 77.27$ & $89.01 \pm 51.62$ & 0.168 & $76.05 \pm 47.42$ & $79.25 \pm 54.31$ & $63.96 \pm 46.76$ & 0.113 & 0.551 & 0.637 \\
\hline \multicolumn{11}{|c|}{ Visuo-motor coordination } \\
\hline \multicolumn{11}{|c|}{ Trail making test (Processing time) } \\
\hline TMT A & $70.51 \pm 33.75$ & $64.75 \pm 26.51$ & $62.99 \pm 32.37$ & 0.408 & $60.07 \pm 31.95$ & $47.94 \pm 18.39$ & $51.45 \pm 23.12$ & 0.152 & 0.058 & 0.513 \\
\hline TMT B & $175.69 \pm 104.78$ & $167.11 \pm 101.27$ & $155.10 \pm 105.00$ & 0.143 & $150.31 \pm 84.92$ & $131.44 \pm 84.31$ & $123.53 \pm 86.10$ & 0.116 & 0.492 & 0.722 \\
\hline \multicolumn{11}{|l|}{ Executive/Frontal } \\
\hline Go/no GO & $11.67 \pm 9.82$ & $15.75 \pm 7.46$ & $17.17 \pm 5.72$ & 0.311 & $16.71 \pm 5.84$ & $19.29 \pm 1.27$ & $19.00 \pm 2.18$ & 0.237 & 0.258 & 0.619 \\
\hline \multicolumn{11}{|l|}{$\begin{array}{r}\text { COWAT } \\
\text { (Fluency) }\end{array}$} \\
\hline Semantic word A & $9.50 \pm 5.62$ & $11.00 \pm 5.43$ & $10.58 \pm 5.05$ & 0.178 & $12.50 \pm 4.36$ & $13.43 \pm 4.91$ & $13.07 \pm 5.00$ & 0.208 & 0.687 & 0.572 \\
\hline Semantic word B & $9.50 \pm 5.68$ & $10.67 \pm 4.60$ & $9.67 \pm 6.02$ & 0.387 & $13.64 \pm 6.30$ & $13.93 \pm 6.09$ & $14.00 \pm 4.91$ & 0.583 & 0.507 & 0.063 \\
\hline \multicolumn{11}{|l|}{ Language } \\
\hline K-BNT & $9.33 \pm 3.94$ & $10.67 \pm 3.63$ & $11.08 \pm 3.26$ & 0.011 & $10.50 \pm 2.95$ & $11.36 \pm 2.87$ & $11.50 \pm 3.32$ & 0.045 & 0.816 & 0.813 \\
\hline
\end{tabular}

Data are mean \pm standard deviation

Inter-p-value: Real vs Sham RS-tDCS

Intra-p-value: Within Sham RS-tDCS group or Real RS-tDCS group

Statistical significance was defined as $p<0.05$

a) RM-ANOVA b) ANCOVA

RS-tDCS: Remotely supervised-trans cranial direct current stimulation CT: Cognitive training K-DRS-2: Korean version of the Dementia Rating Scale-2 TMT: Trail making test COWAT: Controlled oral word association test K-BNT: Korean-Boston naming test

\section{Protocol adherence rate}

Two participants were excluded from the final analysis due to improper application; in both cases, the tDCS device turned off during stimulation because it was not charged in advance. Due to these incidents, the 2 participants only completed 15 and 16 treatment sessions, respectively, which did not meet the target criterion of 17 sessions. Of the 28 participants who continued to the final session, 26 participants completed the intervention, and 551 sessions were successfully completed out of 560 planned sessions. The adherence rate was $98.4 \%$.

\section{Secondary outcomes}

There were no significant differences in the between-group comparison for all items (Table 2). In the within-group comparison, the Stroop test and K-BNT showed significant improvement in both groups, but TMT, Go/no GO, and COWAT-semantic showed no statistically significant difference in groups (Table 2).

\section{Discussion}

To the best of our knowledge, this study is the first to investigate the feasibility and effectiveness of combined RS-tDCS and CT for patients with chronic stroke ( $\geq 6$ months onset). We attempted to show that application of tDCS with home-based CT in patients with stroke is more effective at improving cognitive impairment than with home-based CT alone. In addition, we aimed to demonstrate that the application of RS-tDCS is safe and feasible and propose modification of the environment, tools, and protocol for RS-tDCS.

The findings of this study showed that performing RS-tDCS plus CT for patients with stroke associated with cognitive dysfunction, especially moderate degree, has the potential to augment the effects of CT. Although the difference in K-MoCA score was not statistically significant in the between-group comparison, the fact that only the real group (especially those with moderate cognitive 
dysfunction) showed significant improvement in K-MoCA score in the within-group comparison, suggests that RS-tDCS enhances the effect of CT. In addition, the protocol in this study showed that RS-tDCS could be applied correctly and safely.

\section{Effects on cognitive impairment}

Regarding the K-MoCA, which reflects general cognitive function, the real group showed significant improvement, whereas the sham group did not show any significant improvement in the within-group comparison. In addition, the results of the sub-territory cognitive function test of both groups were similar in each territory in the within-group comparison.

In this study, K-MoCA was more important than other cognitive function tests. The DLPFC, the target area in this study, is a multicognition area. This means that it could have a greater effect on overall cognitive function $[2,7,29]$ than on any single sub-territory, and this could be reflected in the outcomes for K-MoCA, which tests overall cognitive function. Therefore, significant improvement was observed in the K-MoCA evaluation for the real group, whereas no significant improvement was observed in the evaluation of other subterritories of cognitive function.

tDCS has the advantage of easy add-on to the conventional rehabilitation training $[5,15]$. It has been studied that better intervention for stroke recovery is a combination of techniques to maximize neuroplasticity [4]. In a recent tDCS meta-analysis, when tDCS was addedon to motor-related training, motor performance was improved [30]. Furthermore, home-based tDCS combined with occupational therapy improved motor function [31]. tDCS added-on to CT for cognitive improvement would maximize neuroplasticity and is expected to show a greater effect [4], since tDCS shows an additive effect with motor-related rehabilitation treatment. [5]

There was a particularly large improvement in the participant group with moderate cognitive impairment (K-MoCA: 10-17) (Fig. 4). Similarly, a previous study found that tDCS significantly improved visual short-term memory performance in a low performer rather than a high performer, through a mechanism that promotes achievement of maximum capacity [32]. We can surmise that tDCS might show a greater effect in participants with a certain degree of cognitive dysfunction; however, further research is needed to elaborate on these findings. (Table 1). In particular, in this study of RS-tDCS, tDCS seemed to have better effect when targeting patients with moderate cognitive decline (K-MoCA 10-17) that are capable of home-based CT.

\section{Feasibility}

Transcranial magnetic stimulation (TMS) and tDCS are frequently discussed and widely used techniques for brain stimulation. Compared with TMS, tDCS is cheaper, safer, easier to apply, and portable [33]. In this study, RS-tDCS was found to be safe and feasible under the proper protocol. Even though 2 out of 28 participants did not receive the required number of tDCS sessions due to unexpected power-off caused by pre-charging mistake, these were not incidents due to unskilled tDCS application or inappropriate stimulation. This can be prevented by adding simple items to the protocol.

Meanwhile, home-use tDCS [15] has the potential for abuse and misuse, increasing the risk of side effects. There is also an ethical debate concerning the domiciliary utilization of tDCS devices and the demand for suitable restrictions [16]. Therefore, an appropriate level of supervision and monitoring is required; RS-tDCS can be considered more suitable for home-based rehabilitation than home-use tDCS.

In addition, RS-tDCS will help future tDCS studies to include individuals in rural areas, who often experience difficulties participating in research.

\section{RS-tDCS protocol details}

The timing of tDCS application has two mechanisms of action. First, in the online mechanism (during stimulation), tDCS induces an ionic concentration shift in the extracellular fluid, which affects the resting membrane potential, causing hypopolarization at the anode and hyperpolarization at the cathode. Second, in the offline mechanism (after stimulation), hyper-communicative activity occurs at the anode and hypo-communicative activity at the cathode via long-term potentiation and long-term depression [34].

In the present study, the online protocol was used; simultaneous application of online tDCS with home-based rehabilitation is convenient for supervision and improving compliance. In a recent meta-analysis, both online and offline tDCS application appeared to have beneficial effects on cognitive function [2]. Depending on the circumstances, tDCS and CT could be administered separately. Although previous offline application of tDCS for more than 30 minutes did not have a significant effect [35], by the long-term 
potentiation mechanism, generalization of the tDCS effect is induced and, even if tDCS is applied offline, it would be able to show the effect [34]. More research is needed regarding the timing of tDCS application to CT. If possible, using a broader window for the tDCS application timing is beneficial for use in home-based rehabilitation because of the increased flexibility.

For patients with stroke, the DLPFC has been highlighted as a promising brain stimulation target, since it is known to be a multipotent target involved in working memory, attention, and executive function. In a recent meta-analysis, anodal tDCS applied to the DLPFC was reported to be more effective for cognition than when applied to other regions $[2,29]$.

Woods reported that a drift of $1-1.5 \mathrm{~cm}$ in the electrode location can greatly alter the electrical field applied to the whole brain [36]. For correct application, a head cap was used to enable simple and precise positioning of the anode over the left DLPFC and the cathode over the right supraorbital region (Fig. 2). Fixation using an electrode band can result in some electrode drift, depending on the location, and because the tightness of the band can affect the positioning, the band needs to be placed by trained persons, which is unsuitable for home-based rehabilitation. Adjusting the electrode position with head cap is advantageous for home-based rehabilitation.

With regard to safety, there were no serious side effects when tDCS was applied at $2 \mathrm{~mA}$ for up to $30 \mathrm{~min}[5,21]$. A previous study reported that tDCS was tolerable even when applied at $4 \mathrm{~mA}$ [37]. Similarly, in the present study, no serious adverse effects reported. Although there was 1 case of redness and 1 case of dizziness, these symptoms improved after monitoring. In terms of the effectiveness, there is a meta-analysis revealed that an increase in current and charge per unit area had a significant beneficial effect on motor function. [38]

Accordingly, it is necessary to consider reducing the electrode size while applying the same current. However, additional research is needed given that it is a home-based application with potential for slight error in the electrode application location and that tDCS is used for cognitive function rather than motor function.

\section{Limitations}

This study has a few limitations. First, the location of the stroke lesion is known to be an important factor in the recovery of cognitive function [39]; participants in both groups did not have the same location or the same lesion size. Second, it was conducted on a small number of subjects. Therefore, there were limitations in the generalization of effects, and the analysis of underlying intrinsic factors such as BDNF and ApoE [28] was not conducted properly. Third, participant questionnaires for the feasibility assessment were not included. Even if accurate evaluation is difficult due to the patient's cognitive decline, a questionnaire on psychological comfort, economic acceptability, and ease of use of the tDCS application is required.

Furthermore, there may be several other sources of variability affecting the use of tDCS that were not analyzed in this study, such as patient motivation [40], individual differences to tDCS intensity[5], and concomitant tasks and medications affecting the outcomes [4, 5]. Moreover, since this study was focused on home-based rehabilitation, there could also be home-derived variability (e.g., caregiver's cognitive function and number of caregivers involved in the supervision).

\section{Conclusions}

Home-based rehabilitation is becoming increasingly important. In this study, RS-tDCS was found to be safe and feasible and a potentially favorable option for home-based cognitive rehabilitation. RS-tDCS has the potential to enhance the effectiveness of homebased CT, especially in patients with a moderate level of cognitive decline.

\section{List Of Abbreviations}

COWAT $=$ Controlled oral word association test

$\mathrm{CT}=$ cognitive training

DLPFC $=$ dorsolateral prefrontal cortex

K-BNT = Korean-Boston Naming Test

K-DRS-2 $=$ Korean version of the Dementia Rating Scale-2 
$\mathrm{K}-\mathrm{MoCA}=$ Korean version of the Montreal Cognitive Assessment

tDCS = transcranial direct current stimulation

TMS = Transcranial magnetic stimulation

$\mathrm{TMT}=$ trail making test

RS-tDCS = remotely supervised-transcranial direct current stimulation

\section{Declarations}

\section{Ethics approval and consent to participate}

This study was approved by the regional Ethics Committee and registered with Clinical Research information Service (KCT0003427). All participants provided informed consent.

\section{Consent for publication}

Not applicable

\section{Availability of data and materials}

The datasets used and/or analysed during the current study are available from the corresponding author on reasonable request.

\section{Competing interests}

The authors declare that they have no competing interests.

\section{Funding}

This research was supported by a grant from the Korea Health Technology R\&D Project through the Korea Health Industry Development Institute, funded by the Ministry of Health and Welfare, Republic of Korea (grant number: HI15C1529) and Samsung Medical Center Grant (PH0018270).

\section{Authors' contributions}

YHK and MHK was conveived and designed the clinical experiments; JYY, MNS, YJJ, DSK, GWK and YHW conducted the clinical testing and analyzed the data; JYY, YHK and MHK prepared and reviewed the manuscript; SHP, JHS, YHK and MHK revised it critically for important intellectual content. YHK and MHK supervised the complete study. All authors read and approved the final manuscript.

\section{Acknowledgments}

The authors thank all members of the Department of Physical Medicine \& Rehabilitation and Translational Research and Clinical Trials Center for Medical Devices, Jeonbuk National University Hospital and researchers at the Neurorehabilitation Neuroplasticity and Imaging Laboratory (http://neuri.or.kr), Department of Physical and Rehabilitation Medicine, Samsung Medical Center, Korea.

\section{Authors information}

${ }^{1}$ Department of Physical Medicine and Rehabilitation, Jeonbuk National University Medical School, Jeonju, Korea ${ }^{2}$ Research Institute of Clinical Medicine of Jeonbuk National University - Biomedical Research Institute of Jeonbuk National University Hospital, Korea ${ }^{3}$ Department of Physical and Rehabilitation Medicine, Center for Prevention and Rehabilitation, Heart Vascular Stroke Institute, Samsung Medical Center, Sungkyunkwan University School of Medicine, Seoul, Republic of Korea ${ }^{4}$ Department of Health Science and Technology, Department of Medical Device Management and Research, Department of Digital Healthcare, SAIHST, Sungkyunkwan University, Seoul, Republic of Korea ${ }^{5}$ Translational Research \& Clinical Trials Center for Medical Devices, Jeonbuk National University Hospital, Republic of Korea

\section{References}


1. Heinemann AW, Linacre JM, Wright BD, Hamilton BB, Granger C. Prediction of rehabilitation outcomes with disability measures. Arch Phys Med Rehabil. 1994;75:133-43. https://doi.org/10.1016/0003-9993(94)90385-9

2. Summers JJ, Kang N, Cauraugh JH. Does transcranial direct current stimulation enhance cognitive and motor functions in the ageing brain? A systematic review and meta-analysis. Ageing Res Rev. 2016;25:42-54. http://dx.doi.org/10.1016/j.arr.2015.11.004

3. Jeon SY, Han SJ. Improvement of the working memory and naming by transcranial direct current stimulation. Ann Rehabil Med. 2012;36:585-95. http://dx.doi.org/10.5535/arm.2012.36.5.585

4. Lefaucheur J-P, Antal A, Ayache SS, Benninger DH, Brunelin J, Cogiamanian F, et al. Evidence-based guidelines on the therapeutic use of transcranial direct current stimulation (tDCS). Clin Neurophysiol. 2017;128:56-92. http://dx.doi.org/10.1016/j.clinph.2016.10.087

5. Shin Y-I, Foerster Á, Nitsche MA, Reprint of: transcranial direct current stimulation (tDCS)-Application in neuropsychology. Neuropsychologia, 2015;74;74-95. http://dx.doi.org/10.1016/j.neuropsychologia.2015.06.021

6. Jo JM, Kim Y-H, Ko M-H, Ohn SH. Enhancing the working memory of stroke patients using tDCS. Am J Physical Med Rehabil. 2009;88:404-9. doi:10.1097/PHM.0b013e3181a0e4cb

7. Draaisma LR, Wessel MJ, Hummel FC. Non-invasive brain stimulation to enhance cognitive rehabilitation after stroke. Neurosci Lett. 2020;719:133678. https://doi.org/10.1016/j.neulet.2018.06.047

8. Ko M-H., Han S-H, Park S-H, Seo J-H, Kim Y-H. Improvement of visual scanning after DC brain polarization of parietal cortex in stroke patients with spatial neglect. Neurosci Lett. 2008;448;171-74. doi:10.1016/j.neulet.2008.10.050

9. Yi YG, Chun MH, Do KH, Sung EJ, Kwon YG, Kim DY. The effect of transcranial direct current stimulation on neglect syndrome in stroke patients. Ann Rehabil Med. 2016;40:223. http://dx.doi.org/10.5535/arm.2016.40.2.223

10. Elsner B, Kugler J, Pohl M, Mehrholz J. Transcranial direct current stimulation (tDCS) for improving aphasia in adults with aphasia after stroke. Cochrane Database Syst Rev. 2019;5:CD009760. doi: 10.1002/14651858.CD009760.pub4.

11. Laver KE, Adey-Wakeling Z, Crotty M, Lannin NA, George S, Sherrington C, Telerehabilitation services for stroke. Cochrane Database Syst Rev. 2020; doi.org/10.1002/14651858.CD010255.pub3

12. O'Brien SR. Trends in inpatient rehabilitation stroke outcomes before and after advent of the prospective payment system: a systematic review. J Neurol Phys Ther. 2010;34:17-23. doi: 10.1097/NPT.0b013e3181cfd3ac

13. Dobrez D, Heinemann AW, Deutsch A, Manheim L, Mallinson T. Impact of Medicare's prospective payment system for inpatient rehabilitation facilities on stroke patient outcomes. Am J Physical Med Rehabil. 2010;89:198-204. doi:

10.1097/PHM.0b013e3181c9fb40

14. van de Ven RM, Murre JMJ, Veltman DJ, Schmand BA. Computer-based cognitive training for executive functions after stroke: A systematic review. Front Human Neurosci. 2016;10:150. doi: 10.3389/fnhum.2016.00150

15. Palm U, Kumpf U, Behler N, Wulf L, Kirsch B, Wörsching J, et al. Home use, remotely supervised, and remotely controlled transcranial direct current stimulation: a systematic review of the available evidence. Neuromodulation 2018;21:323-33. doi: 10.3389/fnhum.2016.00150

16. Wexler A. The practices of do-it-yourself brain stimulation: implications for ethical considerations and regulatory proposals. J Med Ethics 2016;42:211-5. http://dx.doi.org/10.1136/ medethics-2015-102663

17. Charvet L, Shaw M, Dobbs B, Frontario A, Sherman K, Bikson M, et al. Remotely supervised transcranial direct current stimulation increases the benefit of at-home cognitive training in multiple sclerosis. Neuromodulation 2018;21:383-89. doi: 10.1111/ner.12583

18. Dobbs B, Pawlak N, Biagioni M, Agarwal S, Shaw M, Pilloni G, et al. Generalizing remotely supervised transcranial direct current stimulation (tDCS): feasibility and benefit in Parkinson's disease. J Neuroengineer Rehabil. 2018;15:114. https://doi.org/10.1186/s12984-018-0457-9

19. Charvet LE, Kasschau M, Datta A, Knotkova H, Stevens MC, Alonzo A, et al. Remotely-supervised transcranial direct current stimulation (tDCS) for clinical trials: guidelines for technology and protocols. Front Syst Neurosci. 20159:26. doi: $10.3389 /$ fnsys.2015.00026

20. Irazoki E, Contreras-Somoza LM, Toribio-Guzmán JM, Jenaro-Río C, van der Roest H, Franco-Martín MA. Technologies for cognitive training and cognitive rehabilitation for people with mild cognitive impairment and dementia. A systematic review. Front Psychol. 2020;11:648 doi: 10.3389/fpsyg.2020.00648

21. Russo C, Souza Carneiro MI, Bolognini N, Fregni F. Safety review of transcranial direct current stimulation in stroke. Neuromodulation 2017;20:215-22. doi: 10.1111/ner.12574

Page 10/14 
22. Elsner B, Kugler J, Pohl M, Mehrholz J. Transcranial direct current stimulation (tDCS) for improving activities of daily living, and physical and cognitive functioning, in people after stroke. Cochrane Database Syst Rev. 2016; DOI: 10.1111/ner.12574 10.1002/14651858.CD009645.pub3

23. Dong Y, Kumar Sharma V, Poon-Lap Chan B, Venketasubramanian N, Teoh HL, Chee Seong Seet R, et al. The Montreal Cognitive Assessment (MoCA) is superior to the Mini-Mental State Examination (MMSE) for the detection of vascular cognitive impairment after acute stroke. J Neurol Sci. 2010;299:15-8. doi:10.1016/j.jns.2010.08.051

24. Julayanont P, Nasreddine ZS. Montreal Cognitive Assessment (MoCA): Concept and Clinical Review. In Cognitive Screening Instruments. 2017, p. 139-95. doi 10.1007/978-3-319-44775-9_7

25. Chey J, Lee S. Development of the norms for the Korean-Dementia Rating Scale. Korean J Clin Psychol. 1997;16:423-33.

26. Kang Y, Na D, Hahn S. Seoul Neuropsychological Screening Battery. Incheon: Human Brain Research \& Consulting Co. 2003.

27. Kim YH, Shin SH, Park SH, Ko MG. Cognitive assessment for patient with brain injury by computerized neuropsychological test. J Korean Acad Rehabil Med. 2001;25:209-16

28. Cheeran B, Talelli P, Mori F, Koch G, Suppa A, Edwards M, et al. A common polymorphism in the brain-derived neurotrophic factor gene (BDNF) modulates human cortical plasticity and the response to rTMS. J Physiol. 2008;586:5717-25. doi: 10.1113/jphysiol.2008.159905

29. Brunoni AR, Vanderhasselt M-A. Working memory improvement with non-invasive brain stimulation of the dorsolateral prefrontal cortex: a systematic review and meta-analysis. Brain Cogn. 2014;86:1-9. http://dx.doi.org/10.1016/j.bandc.2014.01.008

30. Marquez J, van Vliet P, McElduff P, Lagopoulos J, Parsons M. Transcranial direct current stimulation (tDCS): does it have merit in stroke rehabilitation? A systematic review. Int J Stroke 2015;10;306-16. doi: 10.1111/ijs.12169

31. Mortensen J, Figlewski K, Andersen H, Combined transcranial direct current stimulation and home-based occupational therapy for upper limb motor impairment following intracerebral hemorrhage: a double-blind randomized controlled trial. Disabil Rehabil. 2016; 38:637-43. http://dx.doi.org/10.3109/09638288.2015.1055379

32. Tseng P, Hsu TY, Chang C-F, Tzeng OJL, Hung DL, Muggleton NG, et al. Unleashing potential: transcranial direct current stimulation over the right posterior parietal cortex improves change detection in low-performing individuals. J Neurosci. 2012;32:10554-61. DOI:10.1523/JNEUROSCI.0362-12.2012

33. Zaghi S, Heine N, Fregni F. Brain stimulation for the treatment of pain: a review of costs, clinical effects, and mechanisms of treatment for three different central neuromodulatory approaches. J Pain Manag. 2009;2:339-52.

34. Stagg CJ, Nitsche MA. Physiological basis of transcranial direct current stimulation. Neuroscientist 2011;17:37-53. doi: $10.1177 / 1073858410386614$

35. Molero-Chamizo A, Alameda Bailén JR, Garrido Béjar T, López MG, Rodríguez IJ, Lérida GC, et al. Poststimulation time intervaldependent effects of motor cortex anodal tDCS on reaction-time task performance. Cogn Affect Behav Neurosci. 2018;8:167-75 https://doi.org/10.3758/s13415-018-0561-0

36. Woods AJ, Bryant V, Sacchetti D, Gervits F, Hamilton R. Effects of electrode drift in transcranial direct current stimulation. Brain Stimul. 2015;8:515-9. http://dx.doi.org/10.1016/j.brs.2014.12.007

37. Chhatbarn PY, Chen R, Deardorff R, Dellenbach B, Kautz SA, George MS, et al. Safety and tolerability of transcranial direct current stimulation to stroke patients-A phase I current escalation study. Brain Stimul. 2017;10:553-9.

http://dx.doi.org/10.1016/j.brs.2017.02.007

38. Chhatbar PY, Ramakrishnan V, Kautz S, George MS, Adams RJ, Feng W. Transcranial direct current stimulation post-stroke upper extremity motor recovery studies exhibit a dose-response relationship. Brain Stimul. 2016;9;16-26. http://dx.doi.org/10.1016/j.brs.2015.09.002

39. Ramsey L, Siegel JS, Lang CE, Strube M, Shulman GL, Corbetta M. Behavioural clusters and predictors of performance during recovery from stroke. Nature Human Behav. 2017;1:1-10. doi: 10.1038/s41562-016-0038

40. Katz B, Au J, Buschkuehl M, Abagis T, Zabel C, Jaeggi SM, et al. Individual differences and long-term consequences of tDCSaugmented cognitive training. J Cogn Neurosci. 2017;29:1498-508. doi:10.1162/jocn_a_01115

\section{Figures}




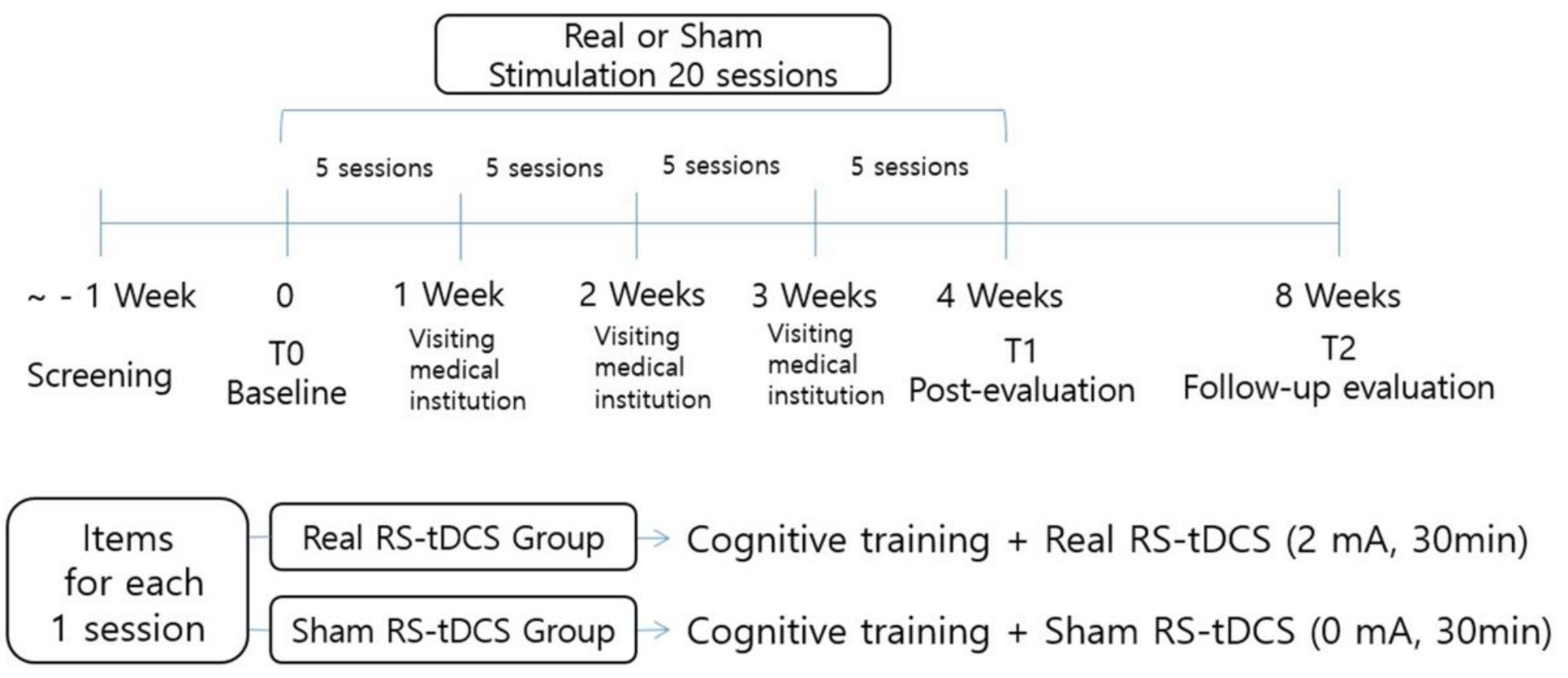

\section{Figure 1}

Study design Computerized CT was performed in both the real and sham groups, and the only differences between them was the application of tDCS stimulation. In each session, CT and tDCS stimulation (real or sham) were applied simultaneously for 30 min. A total of 3 cognitive function tests were conducted: before (T0) and after (T1), and at 4 weeks after (T2) the 20 sessions.

A)

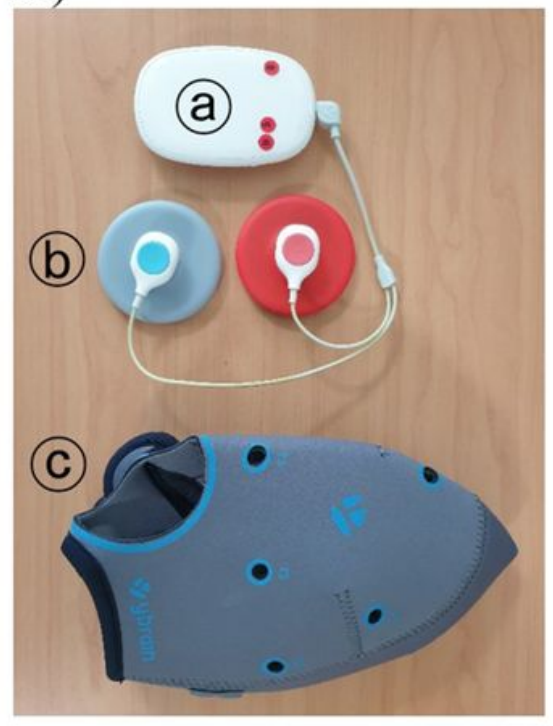

B)

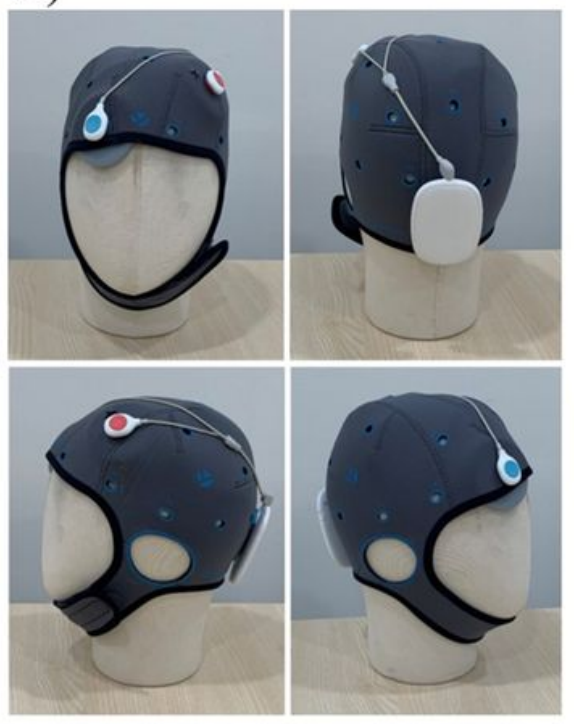

C)

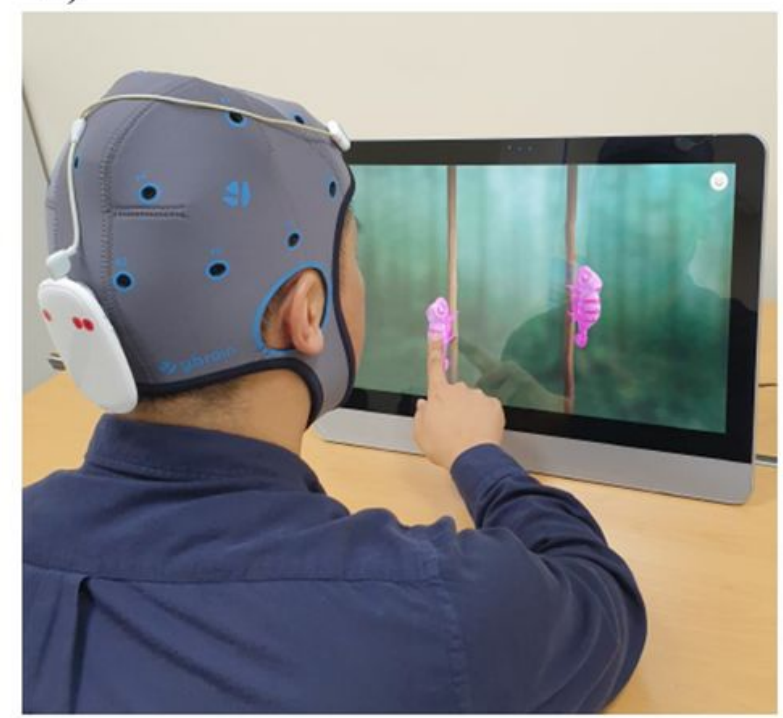

Figure 2

Application of RS-tDCS equipment (Mindd Stim®, Ybrain Inc., Korea) A) Components of RS-tDCS $₫$ Module: source of electrical stimulation, a signal is transmitted to the electrode along the cable. $\nabla$ Electrode: the anode is red, the cathode is blue, and the module is connected by the cable. The diameter of the electrode is $6 \mathrm{~cm}$ (area $28.3 \mathrm{~cm}^{2}$ ). $\nabla$ Head cap: the position of the electrode can be personalized. The fixing hole where the electrode is fixed can be located anywhere on the head cap (by perforating a hole). The chin strap is integrated with the head cap to secure the head cap in a stable position [18]. B) Location of electrode application and head cap wearing with the tDCS module: the anode is placed on the DLPFC, and the cathode is placed on the right supraorbital area. C) Computerized $\operatorname{cog}$ CT with RS-tDCS: the computerized CT program (ComcogTM v.2.6, Neofact, Seoul, Korea, 2018) and RS-tDCS are applied for 30 min simultaneously. 


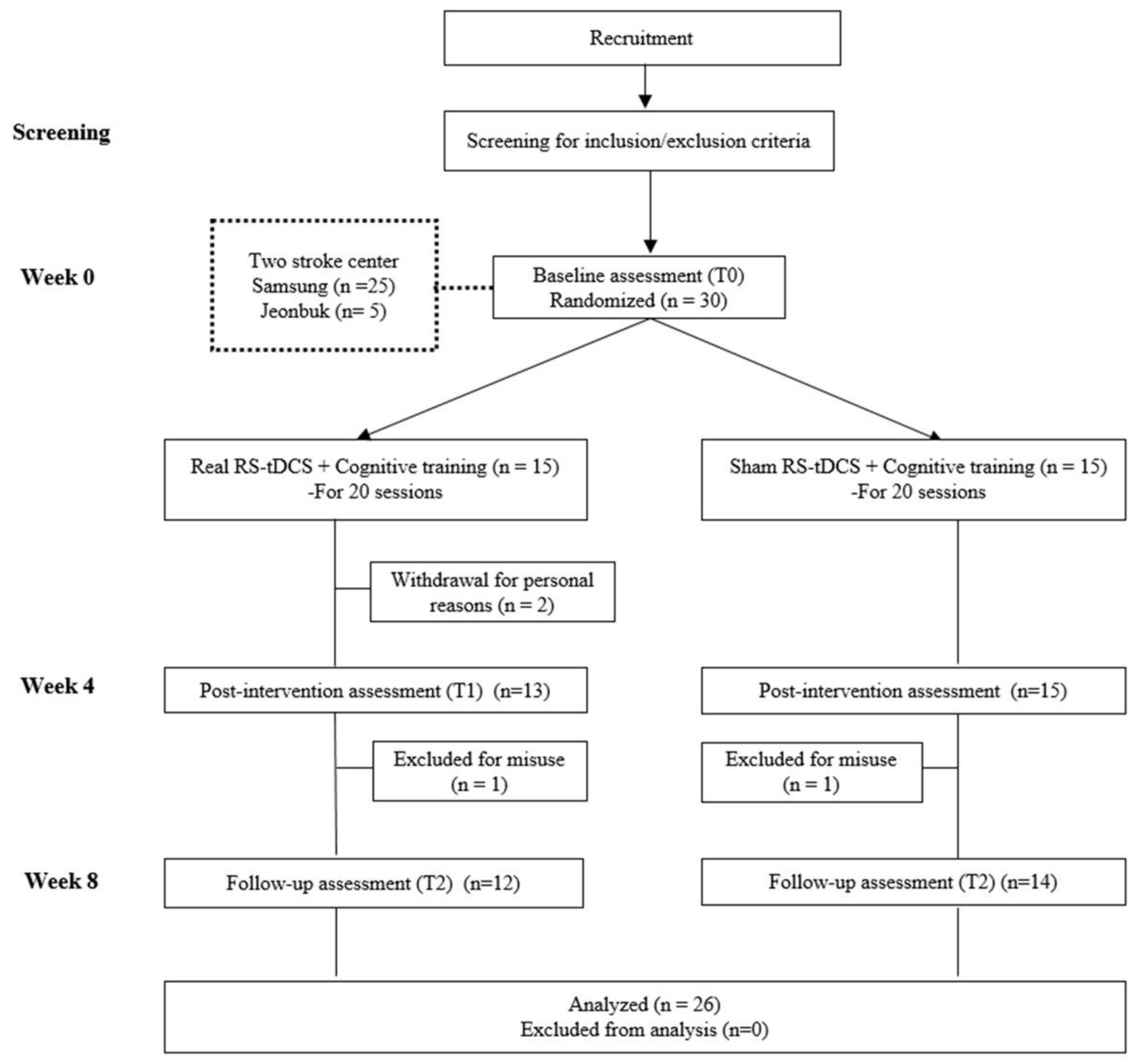

Figure 3

Study flow diagram This study recruited a total of 30 subjects, and 26 of these were evaluated for cognitive function.

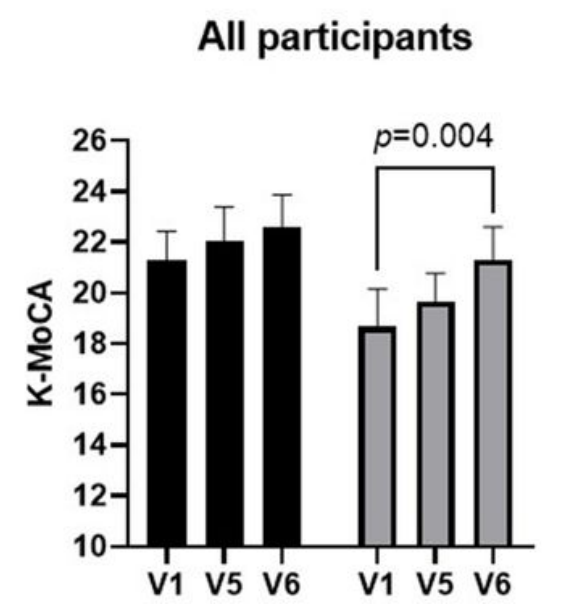

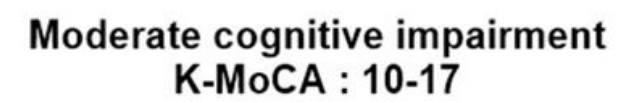

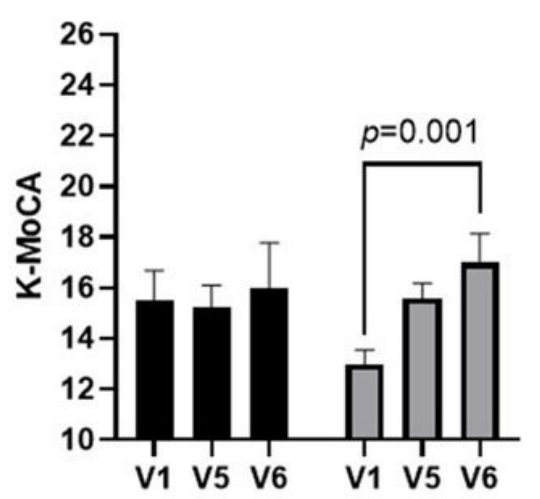

Mild cognitive impairement K-MoCA : 18-25

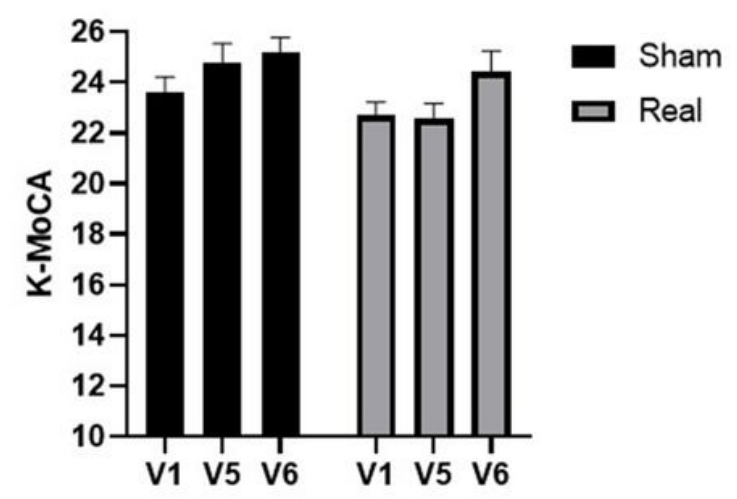

Figure 4 
K-MoCA score for each group according to the level of cognitive function MoCA score was divided into moderate cognitive impairment and mild cognitive impairment. A) K-MoCA score changes of all participants are shown. In the within-group comparison, a statistically significant improvement in the K-MoCA score is observed in the real group but not in the sham group (intra-p-value: Real [p=0.004] vs. Sham [p=0.132]). B) In the within-group comparison of the moderate cognitive impairment group (K-MoCA: 10-17), a statistically significant improvement in the K-MoCA score is observed in the real group but not in the sham group (intra-p-value: Real [ $p=0.001]$ vs. Sham [p=0.835]). C) In the within-group comparison of the mild cognitive impairment group (K-MoCA: 18-25), there is no statistically significant difference in both the real and sham groups (intra-p-value: Real $[p=0.060]$ vs. Sham $[p=0.064]$ ). In the comparison between the two groups (between-group comparison), there is no statistically significant difference in all analyses. Statistical significance was defined as $p<0.05$ 